\title{
Synthesis and Optical Properties of Cubic Gold Nanoframes
}

\author{
Leslie Au', Yeechi Chen ${ }^{1}$, Fei Zhou², Pedro H. C. Camargo ${ }^{3}$, Byungkwon Lim³ ${ }^{3}$ Zhi-Yuan Li², David S. Ginger ${ }^{1}$, \\ and Younan $\mathrm{Xia}^{3}(\bowtie)$ \\ ${ }^{1}$ Department of Chemistry, University of Washington, Seattle, Washington 98195, USA \\ ${ }^{2}$ Institute of Physics, Chinese Academy of Sciences, Beijing 100080, China \\ ${ }^{3}$ Department of Biomedical Engineering, Washington University, St. Louis, Missouri 63130, USA \\ Received: 20 September 2008/ Revised: 14 October 2008/ Accepted: 17 October 2008 \\ CTsinghua Press and Springer-Verlag 2008. This article is published with open access at Springerlink.com
}

\begin{abstract}
This paper describes a facile method of preparing cubic Au nanoframes with open structures via the galvanic replacement reaction between $\mathrm{Ag}$ nanocubes and $\mathrm{AuCl}_{2}{ }^{-}$. A mechanistic study of the reaction revealed that the formation of $\mathrm{Au}$ nanoframes relies on the diffusion of both $\mathrm{Au}$ and $\mathrm{Ag}$ atoms. The effect of the edge length and ridge thickness of the nanoframes on the localized surface plasmon resonance peak was explored by a combination of discrete dipole approximation calculations and single nanoparticle spectroscopy. With their hollow and open structures, the Au nanoframes represent a novel class of substrates for applications including surface plasmonics and surface-enhanced Raman scattering.
\end{abstract}

\section{KEYWORDS}

Gold nanostructures, galvanic replacement, hollow nanostructures, localized surface plasmon resonance, surface-enhanced Raman scattering

Hollow nanostructures of noble metals (e.g., Au, $\mathrm{Pt}$, and $\mathrm{Pd}$ ) have gained attention in recent years for a variety of applications including catalysis [1], optical sensing [2], drug delivery [3], biomedical imaging [4-6], and photothermal therapy [7-10] due to their tunable optical properties and large surface areas. Among various synthetic approaches, the galvanic replacement reaction represents the most versatile route to bimetallic hollow nanostructures [1, 11-16]. Bimetallic hollow nanostructures have been synthesized by reacting Ag nanostructures (or templates) with a salt precursor containing a less reactive metal such as $\mathrm{Au}, \mathrm{Pt}$ or $\mathrm{Pd}$. In particular, the replacement reaction between $\mathrm{Ag}$ nanocubes and
$\mathrm{AuCl}_{4}{ }^{-}$(Eq. (1)) has been extensively explored as a robust method for generating hollow nanostructures in the form of nanoboxes and nanocages [17].

$$
3 \mathrm{Ag}(\mathrm{s})+\mathrm{AuCl}_{4}^{-}(\mathrm{aq}) \rightarrow 3 \mathrm{AgCl}(\mathrm{s})+\mathrm{Au}(\mathrm{s})+\mathrm{Cl}^{-}(\mathrm{aq})
$$

The wall thickness and porosity of these nanostructures are determined by the amount of $\mathrm{AuCl}_{4}{ }^{-}$added to the reaction system. In practice, such control can be easily achieved by titrating Ag nanocubes with different volumes of an aqueous $\mathrm{AuCl}_{4}{ }^{-}$solution.

Recently, several methods have been utilized to further control the porosity and wall thickness of these nanostructures. For example, when the corners of the Ag nanocubes were truncated before undergoing the

Address correspondence to xia@biomed.wustl.edu 
replacement reaction, pores formed selectively at the corners of the cubic nanostructures [18]. Additionally, a wet etchant such as $\mathrm{Fe}\left(\mathrm{NO}_{3}\right)_{3}$ or $\mathrm{NH}_{4} \mathrm{OH}$ can be used for the selective removal of $\mathrm{Ag}$ from the $\mathrm{Au}^{-}$ Ag alloy nanostructures after thin layers of Au have been deposited onto the surfaces of Ag nanocubes [19]. This procedure reduced the wall thickness and caused pores to form on the side faces. At a certain point of the reaction, the pores on each side face were able to coalesce into a single large hole, which led to the formation of a cubic nanoframe, a structure not previously achieved with $\mathrm{AuCl}_{4}{ }^{-}$alone. However, if this specific point was passed during the synthesis, the Au nanoframe broke into small pieces because the ridges became too thin and fragile. The Au nanoframes formed via this route were so sensitive to the reaction conditions that the reported yield never exceeded 5\%-10\%. In contrast, when $\mathrm{AuCl}_{2}{ }^{-}$ was employed as a precursor to $\mathrm{Au}$ instead of $\mathrm{AuCl}_{4}^{-}$, nanoboxes with thicker walls could be generated due to the difference in stoichiometry: in the reaction with $\mathrm{AuCl}_{2}^{-}$(Eq. (2)), one Au atom is formed for every Ag atom consumed, whereas in the reaction with $\mathrm{AuCl}_{4}{ }^{-}$ (Eq. (1)), three $\mathrm{Ag}$ atoms are removed per $\mathrm{Au}$ atom formed [20].

$$
\mathrm{Ag}(\mathrm{s})+\mathrm{AuCl}_{2}^{-}(\mathrm{aq}) \rightarrow \mathrm{AgCl}(\mathrm{s})+\mathrm{Au}(\mathrm{s})+\mathrm{Cl}^{-}(\mathrm{aq})
$$

As this new reaction generated one $\mathrm{Au}$ atom for each Ag atom consumed, we postulated it might provide a method to improve the yields of nanoframes by increasing the thickness (and thus robustness) of the ridges that support the nanoframes. The present work confirms this hypothesis: we were able to increase the yield of a typical synthesis to a level $>90 \%$.

Previously, cubic nanoframes have been reported for $\mathrm{Pd}$ and $\mathrm{Fe}$. In the case of $\mathrm{Pd}$, nanocubes were synthesized by reducing $\mathrm{Na}_{2} \mathrm{PdCl}_{4}$ in ethylene glycol (EG), water, and poly(vinylpyrrolidone) (PVP) and then aged in an oxidizing environment that corroded the structures to form nanoframes [21]. The corrosion process required $4 \mathrm{~h}$ and the total time for the synthesis was $28 \mathrm{~h}$. In the case of $\mathrm{Fe}$, nanoframes were synthesized from the thermal decomposition of a $\mathrm{Fe}(\mathrm{II})$-stearate complex in the presence of sodium oleate and oleic acid [22]. This process required a reaction temperature up to $380{ }^{\circ} \mathrm{C}$ and a reaction time of $3 \mathrm{~h}$, and the mechanism has not yet been established due to its complexity. Here we report a facile (with a total reaction time $<45$ min) synthesis of cubic Au nanoframes as well as a mechanism. The galvanic replacement reaction between $\mathrm{Ag}$ nanocubes and $\mathrm{AuCl}_{2}{ }^{-}$produced hollow nanostructures having thicker walls, in comparison to $\mathrm{AuCl}_{4}^{-}$, that were more robust and could sustain the dealloying process, thus allowing for the formation of Au nanoframes in high yields. We have also examined the different stages of the reaction by electron microscopy. Furthermore, we have explored the effect of the dimensional parameters of the $\mathrm{Au}$ nanoframes on their localized surface plasmon resonance (LSPR) properties and demonstrated the use of these nanoframes as a new class of substrates for surface-enhanced Raman scattering (SERS).

The synthesis of $\mathrm{Au}$ nanoframes was based upon the galvanic replacement reaction between Ag nanocubes and $\mathrm{AuCl}_{2}{ }^{-}$. The $\mathrm{Ag}$ nanocubes with an average edge length of $52 \mathrm{~nm} \pm 4 \mathrm{~nm}$ were synthesized by a sulfide-mediated polyol process as described in previous publications [23, 24]. The Ag nanocubes then served as a sacrificial template for the formation of $\mathrm{Au}$ nanoframes. Figure 1 shows scanning electron microscopy (SEM) and transmission electron microscopy (TEM, inset) images of samples obtained at different stages of the galvanic replacement reaction and Fig. 2 shows a schematic that summarizes this process. In the initial stage, Au was deposited on the surface of the Ag cube and a pinhole formed on one of the six side faces, allowing for the exchange of atoms during the pitting process (Fig. 1(a), and step (a) in Fig. 2). Shortly after, the pinhole closed, generating a partially hollow structure (Fig. 1(b), and step (b) in Fig. 2). The interior of the nanostructure continued to hollow out as Ag atoms diffused to the surface of the structure and $\mathrm{Au}$ diffused into the structure, leading to the formation of a seamless Au nanobox with slightly truncated corners (Fig. 1(c), and step (c) in Fig. 2). This shape reconstruction marked the start of dealloying, where the vacancies from the extraction of $\mathrm{Ag}$ atoms caused negative curvatures, resulting in an increase in interfacial area and surface energy for the solid walls [25]. This stress was relieved via an internal Ostwald ripening process and new $\{111\}$ 
facets were formed [26]. Since the relative surface energies of different facets are in the order of $\gamma_{111}<$ $\gamma_{100}<\gamma_{110}$ for a face-centered cubic (fcc) metal [27, $28]$, a truncated box with a larger portion of $\{111\}$ facets should be more stable than a box with sharp corners. The formation of a truncated rather than a perfect cubic shape has also been observed in many other systems $[29,30]$. Concurrent to the replacement reaction (i.e., Figs. 1(a)-(c), and steps (a)-(c) in Fig. 2), the rates of diffusion for $\mathrm{Ag}$ and $\mathrm{Au}$ were accelerated by the relatively high reaction temperature of $100{ }^{\circ} \mathrm{C}$ [31] and led to the subsequent formation of a $\mathrm{Au}-\mathrm{Ag}$ alloy that is more stable than pure $\mathrm{Au}$ or $\mathrm{Ag}$ alone [32]. When more $\mathrm{AuCl}_{2}^{-}$was added, the removal of $\mathrm{Ag}$ and deposition of Au occurred at all sites, and pores appeared at the corners and side faces (Fig. 1(d), and step (d) in Fig. 2). As dealloying continued, the pores on the side faces enlarged while the pores at the corners reduced in size (Fig. 1(e), and step (e) in Fig. 2 ), suggesting that the atoms migrated to the more stable $\{111\}$ facet. Eventually, the pores at the corners were sealed while the pores on all the side faces were enlarged to the maximum size, resulting in the formation of a cubic nanoframe (Fig. 1(f), and step (f) in Fig. 2). This is the critical point for harvesting $\mathrm{Au}$ nanoframes with a yield approaching $100 \%$. When more $\mathrm{AuCl}_{2}{ }^{-}$was added, the ridges became thinner as a result of dealloying of $\mathrm{Ag}$ atoms from the structure (Fig. 1(g), and step (g) in Fig. 2). At a certain point the ridges became too thin to support the open structure, such that the nanoframes fragmented and finally evolved into Au nanoparticles (Fig. 1(h), and step (h) in Fig. 2).

The SEM image in Fig. 3(a) shows Au nanoframes with an average edge length of $63 \mathrm{~nm} \pm 4 \mathrm{~nm}$ and ridge thickness of $19 \mathrm{~nm} \pm 2 \mathrm{~nm}$ that were synthesized under the same conditions as for Fig. 1(f). The elemental composition of these nanoframes was determined to be $89 \% \mathrm{Au}$ and $11 \% \mathrm{Ag}$ by energy-dispersive X-ray spectroscopy (EDX). To better resolve the three-dimensional structure of the nanoframes, the sample was tilted by $45^{\circ}$ as shown in Fig. 3(b). Figure 3(c) shows a TEM image of the nanoframes, from which one can easily resolve the hollow structures and determine the thickness of the ridges. Figure 3(d) shows a high-resolution TEM

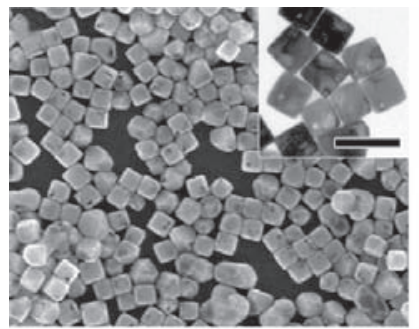

(a)

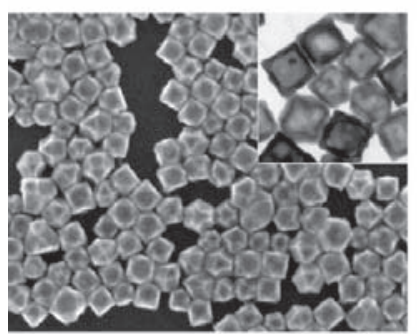

(c)

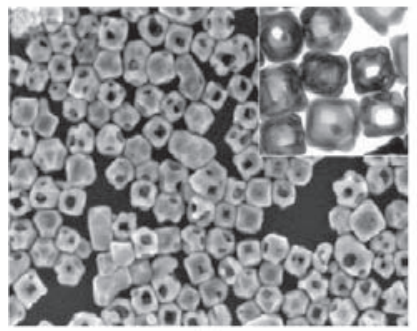

(e)

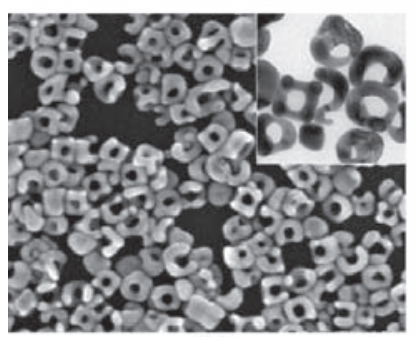

(g)

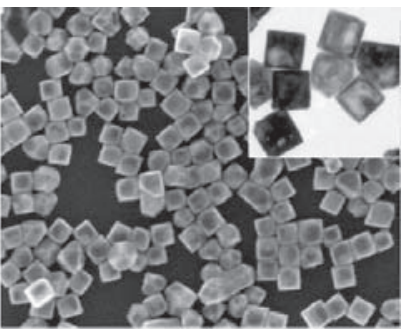

(b)

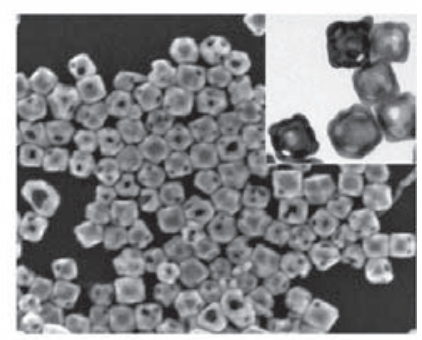

(d)

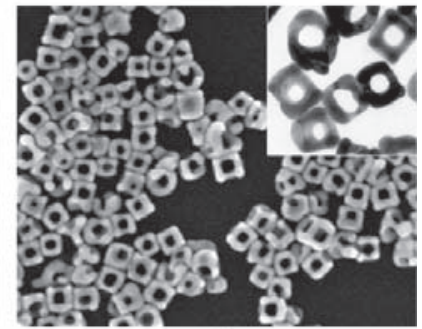

(f)

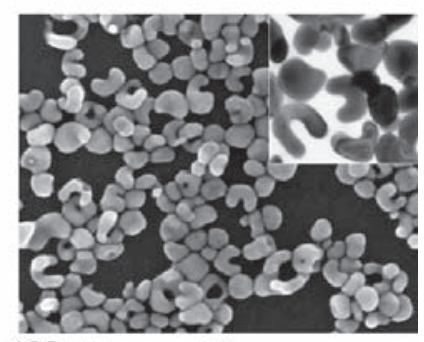

(h)
Figure 1 SEM and TEM (insets) images showing different stages of the galvanic replacement reaction where $\mathrm{Ag}$ nanocubes were titrated with different volumes of $0.2 \mathrm{mmol} / \mathrm{L} \mathrm{AuCl}_{2}{ }^{-}$in $1 \mathrm{~mL}$ increments ranging from (a) $1 \mathrm{~mL}$ to $(\mathrm{h}) 8 \mathrm{~mL}$. The scale bar below the images applies to all SEM images. The scale bar in the inset (a) represents $100 \mathrm{~nm}$ and applies to all TEM images

image of a corner of the nanoframe taken along the [001] zone axis. This image indicates that the atoms are arranged in a highly ordered lattice with a spacing of $2.04 \AA$, which can be indexed to the $\{200\}$ planes of fcc Au. The fast Fourier transform (FFT) pattern revealed that the nanoframe is single crystalline in structure. The spots enclosed by the circle and squares can be indexed to the $\{220\}$ and $\{200\}$ reflections, respectively.

Figure 4(a) summarizes the UV-vis spectra taken

\section{(a) \\ 黛 Springer}




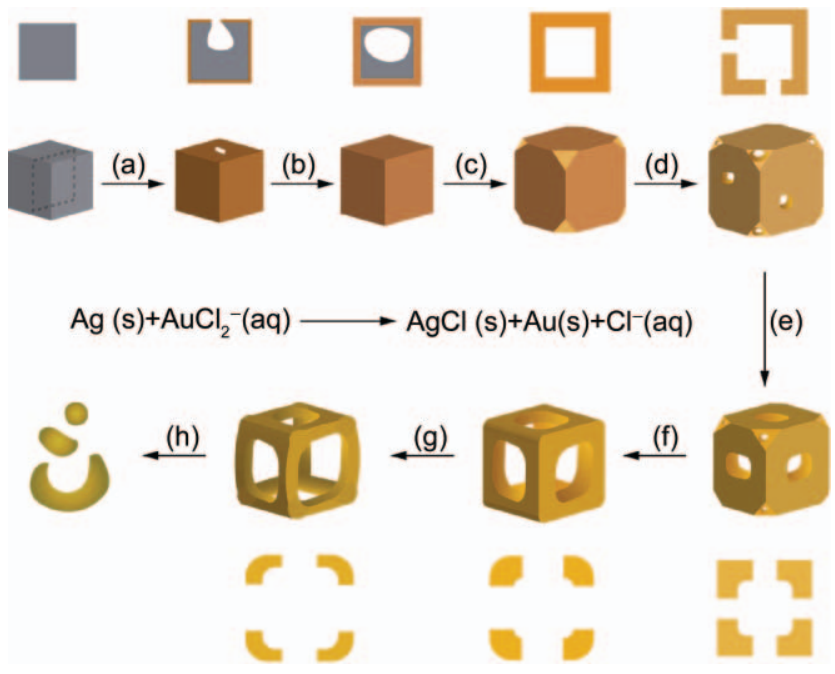

Figure 2 A schematic detailing the mechanism of the galvanic replacement reaction between $\mathrm{Ag}$ nanocubes and $\mathrm{AuCl}_{2}^{-}$. The crosssectional view corresponds to the plane marked by dashed lines. The major steps of the reaction include the following: (a) formation of a pinhole at one of the side faces; (b) continuation of the replacement reaction resulting in a partially hollow structure; (c) development of a seamless nanobox with truncated corners; (d) generation of pores at the corners and side faces by a dealloying process; (e), ( $f$ ) enlargement of pores at the side faces accompanied by shrinkage of pores at the corners via migration of atoms to the corners; ( $g$ ) reduction of the ridge thickness; (h) fragmentation of the nanoframes

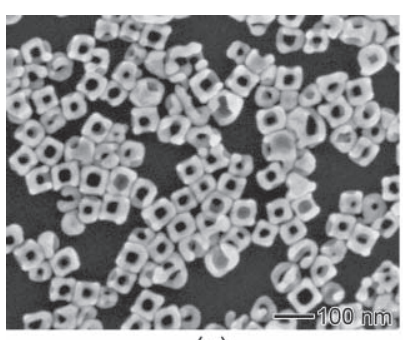

(a)

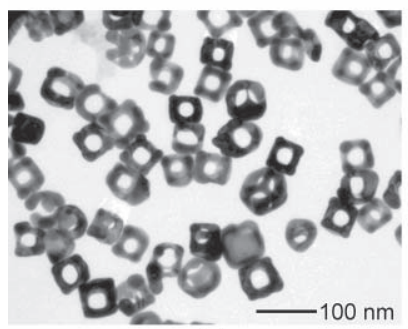

(c)

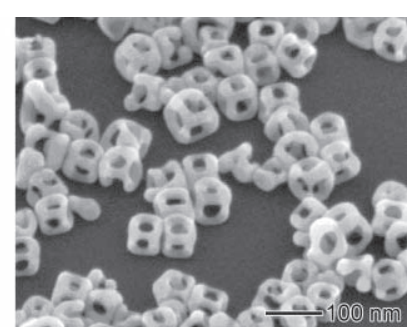

(b)

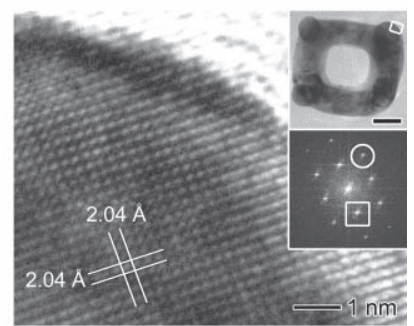

(d)
Figure 3 Electron microscopy characterization of the Au nanoframes prepared by the galvanic replacement reaction between $50 \mu \mathrm{L}$ of $\mathrm{Ag}$ nanocubes and $6 \mathrm{~mL}$ of $0.2 \mathrm{mmol} / \mathrm{L} \mathrm{AuCl}_{2}^{-}$: (a) SEM image of the Au nanoframes and (b) the same sample tilted by $45^{\circ}$; (c) TEM image of the Au nanoframe; (d) high-resolution TEM image of a corner of the Au nanoframe taken from the region as labeled in the inset. The scale bar in the inset represents $20 \mathrm{~nm}$. The lattice spacing of $2.04 \AA$ can be indexed as the $\{200\}$ planes of Au. In the FFT pattern (inset), the spots enclosed by the circle and square can be indexed to the $\{220\}$ and $\{200\}$ reflections, respectively in water of the structures shown in Figs. 1(a)-(f). As more $\mathrm{AuCl}_{2}^{-}$was added, a significant red-shift was observed. The UV-vis spectrum of the Au nanoframes obtained by addition of $6 \mathrm{~mL}$ of $\mathrm{AuCl}_{2}^{-}$solution (Fig. 4(a)) had an LSPR peak around $790 \mathrm{~nm}$. The broadness of the peak may be ascribed to the variations in edge length, ridge thickness, and corner sharpness. Potential applications such as imaging, SERS, and chemical and biological sensing are dependent upon LSPR properties; it is, therefore, important to understand the relationship between such structural variations and LSPR properties in order to effectively tune the plasmon peak. It has been established for $\mathrm{Au}$ or Ag nanostructures of other shapes [5, 23, 3337] that the LSPR property of a nanoframe depends on several factors including the edge length, ridge thickness, corner sharpness, elemental composition, laser orientation, and environment. To determine how these factors, specifically the size and shape, influence the LSPR peak, the scattering spectra of individual nanoframes were calculated in air using the discrete dipole approximation (DDA) method. After reviewing many spectra, we found a correlation between the peak position and the ratio $(R)$ between the outer edge length $(l)$ and the ridge thickness $(t)$. Figure $4(b)$ shows the spectra calculated for nanoframes with an edge length of 57.0, 59.4, 61.7, 64.1, 66.5, and $68.9 \mathrm{~nm}$, having a constant ridge thickness of $19 \mathrm{~nm}$ and a fixed composition of $89 \% \mathrm{Au}$ and $11 \% \mathrm{Ag}$. The nanoframes were assumed to have sharp corners as shown in the inset. As the value of $R$ increased, the peak position was red-shifted while the peak intensity slightly increased. Figure 4(c) shows the DDA-calculated extinction, absorption and scattering spectra for the nanoframe with $R=3.37$, and geometry as shown in the inset.

To confirm the theoretical calculations, nanoframes were deposited on indium tin oxide (ITO)-coated glass substrates and the scattering spectra of single nanoframes were collected in air using an inverted microscope with a transmitted dark-field condenser as previously described [38-41]. The scattering spectra are plotted in Fig. 4(d) along with the SEM images of the corresponding nanoframes in the inset. We observed that the spectra were red-shifted as the $R$ value increased, validating the trend found 


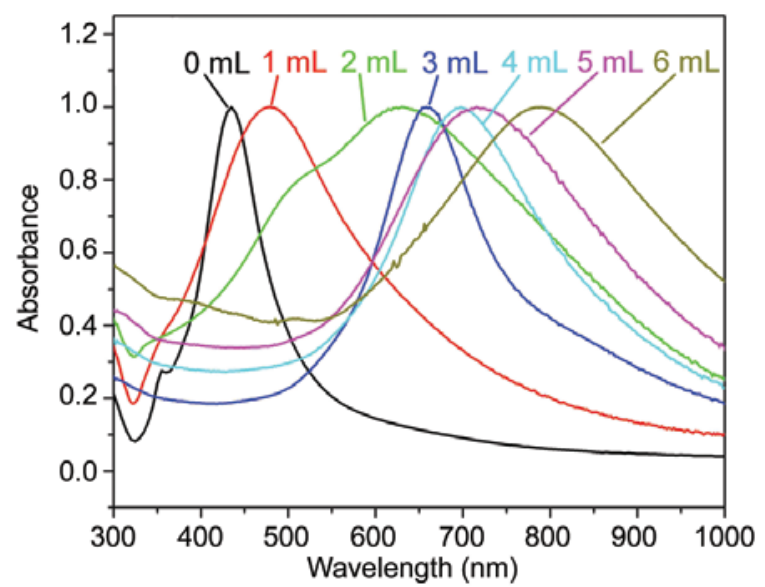

(a)

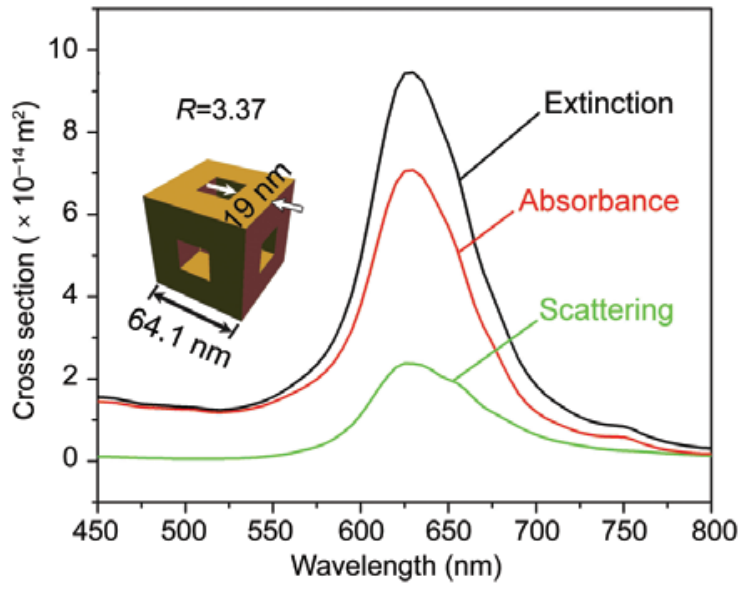

(c)

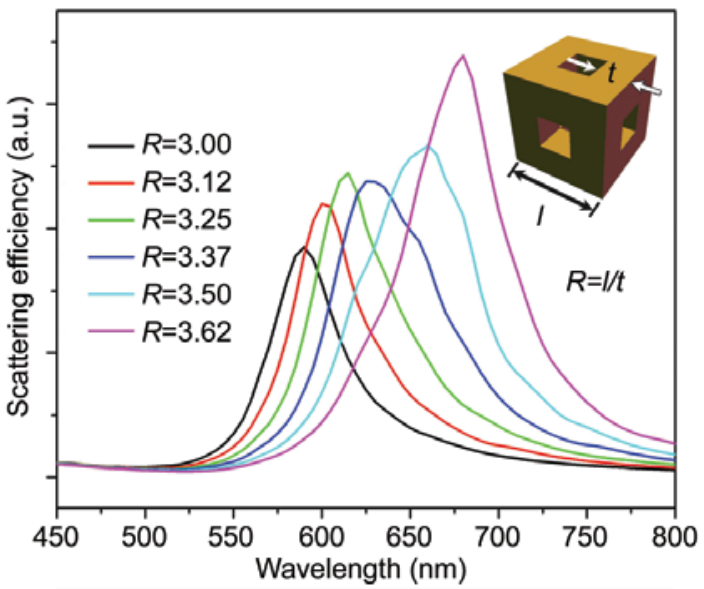

(b)

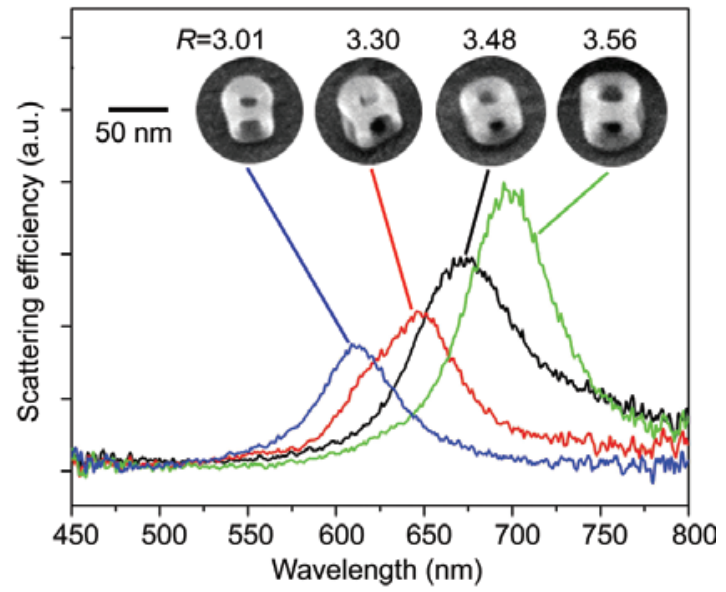

(d)

Figure 4 (a) UV-vis spectra taken from aqueous suspensions of the structures in Figs. 1(a)-(f), which were synthesized by titrating Ag nanocubes with $1 \mathrm{~mL}$ to $6 \mathrm{~mL}$ of $0.1 \mathrm{mmol} / \mathrm{L} \mathrm{AuCl}_{2}^{-}$. (b) DDA-calculated scattering spectra for nanoframes with an edge length of 57.0,59.4,61.8,64.1, 66.5, and $68.9 \mathrm{~nm}$, having a constant ridge thickness of $19 \mathrm{~nm}$, together with a fixed composition of $89 \% \mathrm{Au}$ and $11 \% \mathrm{Ag}$. The inset shows a drawing of the nanoframe used in these calculations, which has both sharp corners and edges. The red-shifts of the plasmon resonance peak increase with increasing ratio $(R)$ between the outer edge length ( () and the ridge thickness $(t)$. (c) DDA-calculated extinction, scattering, and absorption spectra for a nanoframe with $R=3.37$. (d) Scattering spectra of individual Au nanoframes and the SEM images of the corresponding nanoframes. For DDA calculations in (b) and (c), the nanoframes were filled and surrounded by air

by DDA calculations. This result is expected as a decrease in ridge thickness or increase in edge length will increase the charge separation, thus reducing the restoring force for electron oscillation and resulting in red-shifting for the resonance peak. As previously reported for hollow nanospheres, thinner shells have stronger coupling between charges inside and outside of the shell, causing greater charge separation and a further red-shift in peak position [34]. As for the calculated spectra, the peak intensity was higher for larger $R$ values; this trend is also expected, as larger particles scatter more light.

It is worth pointing out that the resonance peaks for the calculated spectra did not completely match the experimental spectra, which can be ascribed to the complexity of the structures. In the ideal situation, a nanoframe has uniform pore size, ridge thickness, and edge length in addition to sharp corners as seen in the inset of Fig. 4(b). From the electron microscopy images in Figs. 3 and 4(d), it is clear that within one nanoframe there are variations in these features which would result in some shifts of the resonance peak. Additionally, it has previously been shown that the substrate can shift the LSPR peak [37]. We expect to observe this substrate effect since the DDA calculations of the nanoframes were 
performed for particles in air and the experimental spectra were collected for particles on an ITO-coated glass substrate in air.

The Au nanoframes can also serve as substrates for SERS-based detection. Due to their tunable LSPR peaks-which can extend into the nearinfrared region - they are ideal candidates for detection of molecules in vivo, as the light source in this region can penetrate deeply into soft tissue. As a demonstration of their SERS application, the Au nanoframes were functionalized with 4-methylbenzenethiol (4-MBT, a common probe molecule for SERS) and their SERS spectrum was recorded in a solution phase setup. Figure 5 shows the SERS spectrum for the Au nanoframes functionalized with 4-MBT (spectrum A) along with the ordinary Raman spectrum of 4-MBT (spectrum B), both obtained with excitation at $785 \mathrm{~nm}$. As illustrated in Fig. 5, utilization of the Au nanoframes led to a significant enhancement in the intensity of the 4-MBT signals compared to the spectrum of neat 4-MBT, indicating that they are good substrates for SERS. However, an accurate estimation of the enhancement factor is limited by the complex morphology of the Au nanoframes, which makes it difficult to precisely determine their surface area and, consequently, the number of 4-MBT molecules contributing to the detected SERS signals.

In conclusion, we have synthesized Au
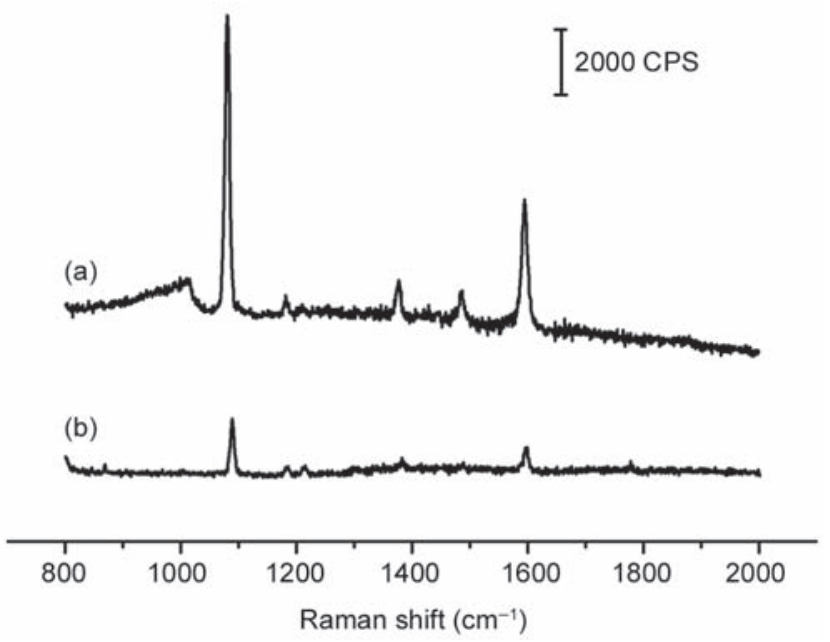

Figure 5 (a) SERS spectrum of Au nanoframes whose surface had been derivatized with 4-MBT; (b) The ordinary Raman spectrum of 4-MBT $(0.1 \mathrm{~mol} / \mathrm{L}$ in $12 \mathrm{~mol} / \mathrm{L}$ aqueous $\mathrm{NaOH}$ ) taken for reference. The measurements were performed in a solution phase with an excitation wavelength of $785 \mathrm{~nm}$ nanoframes with a yield $>90 \%$ through the facile galvanic replacement reaction between $\mathrm{Ag}$ nanocubes and $\mathrm{AuCl}_{2}^{-}$. We have also examined the evolution pathway from $\mathrm{Ag}$ nanocubes to $\mathrm{Au}$ nanoframes by conducting a detailed mechanistic study with the addition of different volumes of $\mathrm{AuCl}_{2}{ }^{-}$solution. We have characterized the effect of the dimensional parameters of the Au nanoframes on the positions of their LSPR peak. Due to their highly open structure, the nanoframes are potentially useful for SERS detection of analytes such as viruses and bacteria with relatively large dimensions.

\section{Experimental}

Synthesis of Ag nanocubes: The Ag nanocubes were prepared using the sulfide-mediated polyol process as described in previous publications [23, 24]. In a typical synthesis, 6 mL EG (J. T. Baker, 9300-03) was preheated to $155{ }^{\circ} \mathrm{C}$ for $1 \mathrm{~h}$ under magnetic stirring. EG solutions containing $3 \mathrm{mmol} / \mathrm{L} \mathrm{Na}_{2} \mathrm{~S}$ (Aldrich, 208043), $0.18 \mathrm{~mol} / \mathrm{L}$ PVP (as calculated in terms of the repeating unit, $M_{\mathrm{w}} \sim 55000$, Aldrich, 856568), and $0.28 \mathrm{~mol} / \mathrm{L} \mathrm{AgNO}_{3}$ (Aldrich, 209139) were prepared. $80 \mu \mathrm{L}$ of the $\mathrm{Na}_{2} \mathrm{~S}$ solution was injected into the hot EG, followed by $1.5 \mathrm{~mL}$ of the PVP solution and then $0.5 \mathrm{~mL}$ of the $\mathrm{AgNO}_{3}$ solution. The reaction underwent color changes from yellow to reddish brown to opaque green-gray with plating on the vial walls. The reaction was completed within $20 \mathrm{~min}$. The reaction solution was diluted with acetone, and the product was isolated by centrifugation. The product was washed twice with deionized water and then collected by centrifugation at $13000 \mathrm{rpm}$ for 5 min and redispersed by brief sonication in $4 \mathrm{~mL}$ of deionized water.

Synthesis of Au nanoframes: The Au nanoframes were prepared using the galvanic replacement reaction between the $\mathrm{Ag}$ nanocubes and $\mathrm{AuCl}_{2}{ }^{-}$. In a typical synthesis, $50 \mu \mathrm{L}$ of $\sim 3.5 \mathrm{nmol} / \mathrm{L} \mathrm{Ag}$ nanocubes was dispersed in $5 \mathrm{~mL}$ of deionized water containing $5 \mathrm{mg}$ PVP in a 50-mL flask under magnetic stirring and then heated to boiling for $10 \mathrm{~min}$. In the meantime, a solution of $0.2 \mathrm{mmol} / \mathrm{L} \mathrm{AuCl}$ (Aldrich, 481130) in saturated aqueous $\mathrm{NaCl}$ was prepared. $\mathrm{AuCl}$ was washed three times with chloroform to 
remove $\mathrm{Au}(\mathrm{III})$ and thoroughly dried in vacuum prior to usage. A specific amount (as indicated in the text) of the resulting $\mathrm{NaCl}$-saturated $\mathrm{AuCl}_{2}^{-}$solution was added to the flask via a syringe pump (Stoelting, KDS-200) at a rate of $45 \mathrm{~mL} / \mathrm{h}$ under magnetic stirring. The solution was heated for another $10 \mathrm{~min}$ until the color of the mixture was stable. Once cooled to room temperature, the sample was washed with saturated $\mathrm{NaCl}$ solution to remove $\mathrm{AgCl}$ and then with water several times to remove PVP and $\mathrm{NaCl}$. The product was then collected by centrifugation at $10000 \mathrm{rpm}$ and redispersed in water. The product was subsequently imaged by SEM and TEM.

Electron microscopy characterization: SEM or TEM samples were prepared by placing a drop of the final product on a silicon wafer or carbon-coated copper grid, respectively, and drying under ambient conditions. SEM images and EDX data were taken using a Sirion XL field-emission microscope operated at an acceleration voltage of $10 \mathrm{kV}$. TEM imaging was performed using a Phillips CM100 microscope operated at $100 \mathrm{kV}$. The UV-vis spectra were obtained using a Varian Cary 50 UV-vis spectrophotometer.

Substrate preparation for optical characterization: ITO-coated glass substrates (Thin Film Devices Inc.) were cleaned by sonication in acetone and then isopropanol for $30 \mathrm{~min}$ in each solvent and then dried under a nitrogen stream. The substrates were then plasma cleaned (Harrick Plasma, PDC$32 \mathrm{G}$ ) for 3 min with an applied power of $18 \mathrm{~W}$ before immersing in a solution of $1 \mathrm{mmol} / \mathrm{L} \mathrm{3}$-mercapto propyltrimethoxysilane in ethanol for $4 \mathrm{~h}$. The substrates were then rinsed with ethanol and cured at $90{ }^{\circ} \mathrm{C}$ under nitrogen for another $4 \mathrm{~h}$, and thereafter stored at room temperature. Before deposition, the stock solution of $\mathrm{Au}$ nanoframes was sonicated for 30 s. The nanoframes were diluted 20X with water. The Au nanoframes were allowed to bind onto the surface for approximately $2 \mathrm{~min}$, giving a final particle density of $\sim 10$ particles per $100 \mu \mathrm{m}^{2}$. After depositing, the excess particles were extensively rinsed off the surface, and the substrate was soaked in water for 20 min to remove excess PVP, rinsed in water, and finally dried by a nitrogen stream. Scattering spectra were taken with an inverted microscope (Nikon, TE2000) with a transmitted dark-field condenser and
75X effective magnification as previously described [38-40]. After optical measurements, the same Au nanoframes were identified and imaged under SEM with the assistance of registration marks [35, 36, 40, 41]. This allows us to correlate the optical spectra to the structural and dimensional information obtained from SEM imaging.

Surface-enhanced Raman scattering measurements: SERS spectra were obtained from aqueous solutions using a Renishaw in via Raman spectrometer coupled to a Leica DMIRB inverted optical microscope. A diode laser with an excitation wavelength $785 \mathrm{~nm}$ was used at a power of $4.5 \mathrm{~mW}$. The backscattered Raman signals were collected on a charge-coupled device detector thermoelectrically cooled to $-60{ }^{\circ} \mathrm{C}$. Sample cells were constructed by trimming the bottoms of plastic micro-centrifuge tubes and affixing the top portions to glass cover slips of thickness between 0.13 and $0.17 \mathrm{~mm}$. Solutions for SERS measurements were prepared by dispersing the $\mathrm{Au}$ nanoframes in $1.5 \mathrm{~mL}$ of $5 \mathrm{mmol} / \mathrm{L}$ ethanolic solution of 4-MBT for $3 \mathrm{~h}$ prior to the SERS measurements, followed by successive rounds of centrifugation and washing with ethanol to remove excess 4-MBT. The sample was dried under ambient conditions to remove ethanol and then redispersed in $100 \mu \mathrm{L}$ of water. The ordinary Raman spectrum of 4-MBT was obtained from a $0.1 \mathrm{~mol} / \mathrm{L}$ solution in $12 \mathrm{~mol} / \mathrm{L}$ aqueous $\mathrm{NaOH}$. To ensure that the focal volume was held constant, a motorized Z-stage with an accuracy of $0.1 \mu \mathrm{m}$ was used to control the focal depth. This was done by focusing the interface between the glass and the solution, and then using the motorized stage to adjust the focal plane $50 \mu \mathrm{m}$ into the solution. Scattering spectra were recorded from 800 to $2000 \mathrm{~cm}^{-1}$ by using a grating with 1200 lines per millimeter, a spot size of approximately 1.6 $\mu \mathrm{m}$, and $30 \mathrm{~s}$ accumulation time.

\section{Acknowledgements}

This work was supported in part by a Director's Pioneer Award from the NIH (5DPOD000798, Y.X.), the Air Force Office of Scientific Research (D.S.G. and Y.C.), the National Science Foundation (DMR 0520567, D.S.G. and Y.C.), and the National Natural 
Science Foundation of China (10525419, 60736041, and 10874238, Z.Y.L.). L.A. thanks the Center for Nanotechnology at the UW for an IGERT Fellowship funded by the NSF and NCI.

\section{References}

[1] Kim, S. W.; Kim, M.; Lee, W. Y.; Hyeon, T. Fabrication of hollow palladium spheres and their successful application to the recyclable heterogeneous catalyst for Suzuki coupling reactions. J. Am. Chem. Soc. 2002, 124, 76427643.

[2] Sun, Y.; Xia, Y. Increased sensitivity of surface plasmon resonance of gold nanoshells compared to that of gold solid colloids in response to environmental changes. Anal. Chem. 2002, 74, 5297-5305.

[3] Portney, N. G.; Ozkan, M. Nano-oncology: Drug delivery, imaging, and sensing. Anal. Bioanal. Chem. 2006, 384, 620-630.

[4] Chen, J.; Saeki, F.; Wiley, B. J.; Cang, H.; Cobb, M. J.; Li, Z. -Y.; Au, L.; Zhang, H.; Kimmey, M. B.; Li, X.; Xia, Y. Gold nanocages: Bioconjugation and their potential use as optical imaging contrast agents. Nano Lett. 2005, 5, 473-477.

[5] Chen, J.; Wiley, B. J.; Li, Z. -Y.; Campbell, D.; Saeki, F.; Cang, H.; Au, L.; Lee, J.; Li, X.; Xia, Y. Gold nanocages: Engineering their structure for biomedical applications. Adv. Mater. 2005, 17, 2255-2261.

[6] Cang, H.; Sun, T.; Li, Z. -Y.; Chen, J.; Wiley, B. J.; Xia, Y.; Li, X. Gold nanocages as contrast agents for spectroscopic and conventional optical coherence tomography. Opt. Lett. 2005, 30, 3048-3050.

[7] Loo, C.; Lin, A.; Hirsch, L.; Lee, M. H.; Barton, J.; Halas, N.; West, J.; Drezek, R. Nanoshell-enabled photonics-based imaging and therapy of cancer. Technol. Cancer Res. Treat. 2004, 3, 33-40.

[8] Chen, J.; Wang, D.; Xi, J.; Au, L.; Siekkinen, A.; Warsen, A.; Li, Z. -Y.; Zhang, H.; Xia, Y.; Li, X. Immuno gold nanocages with tailored optical properties for targeted photothermal destruction of cancer cells. Nano Lett. 2007, 7, 1318-1322.

[9] Hirsch, L. R.; Gobin, A. M.; Lowery, A. R.; Tam, F.; Drezek, R. A.; Halas, N. J.; West, J. L. Metal nanoshells. Ann. Biomed. Eng. 2006, 34, 15-22.

[10] Au, L.; Zheng, D.; Zhou, F.; Li, Z. -Y.; Li, X.; Xia, Y. A quantitative study on the photothermal effect of immuno gold nanocages targeted to breast cancer cells. ACS Nano 2008, 2, 1645-1652.

[11] Sun, Y.; Xia, Y. Shape-controlled synthesis of gold and silver nanoparticles. Science 2002, 298, 2176-2179.

[12] Sun, Y.; Mayers, B.; Xia, Y. Metal nanostructures with hollow interiors. Adv. Mater. 2003, 15, 641-646.

[13] Wiley, B. J.; Sun, Y.; Chen, J.; Cang, H.; Li, Z. -Y.; Li, $X$.; Xia, Y. Shape-controlled synthesis of silver and gold nanostructures. MRS Bull. 2005, 30, 356-361.

[14] Yang, J.; Lee, J. Y.; Too, H. P. Core-shell Ag-Au nanoparticles from replacement reaction in organic medium. J. Phys. Chem. B 2005, 109, 19208-19212.

[15] Chen, J.; McLellan, J. M.; Siekkinen, A.; Xiong, Y.; Li, Z. -Y.; Xia, Y. Facile synthesis of gold-silver nanocages with controllable pores on the surface. J. Am. Chem. Soc. 2006, 128, 14776-14777.

[16] Yin, Y.; Erdonmez, C.; Aloni, S.; Alivisatos, A. P. Faceting of nanocrystals during chemical transformation: From solid silver spheres to hollow gold octahedra. J. Am Chem. Soc. 2006, 128, 12671-12673.

[17] Sun, Y.; Xia, Y. Mechanistic study on the replacement reaction between silver nanostructures and chloroauric acid in aqueous medium. J. Am. Chem. Soc. 2004, 126, 3892-3901.

[18] Chen, J.; Wiley, B. J.; McLellan, J.; Xiong, Y.; Li, Z. -Y.; $\mathrm{Xia}, \mathrm{Y}$. Optical properties of $\mathrm{Pd}-\mathrm{Ag}$ and $\mathrm{Pt}-\mathrm{Ag}$ nanoboxes synthesized via galvanic replacement reactions. Nano Lett. 2005, 5, 2058-2062.

[19] Lu, X.; Au, L.; McLellan, J.; Li, Z. -Y.; Marquez, M.; Xia, $Y$. Fabrication of cubic nanocages and nanoframes by dealloying Au/Ag alloy nanoboxes with an aqueous etchant based on $\mathrm{Fe}\left(\mathrm{NO}_{3}\right)_{3}$ or $\mathrm{NH}_{4} \mathrm{OH}$. Nano Lett. 2007, 7, 1764-1767.

[20] Au, L.; Lu, X.; Xia, Y. A comparative study of galvanic replacement reactions involving $\mathrm{Ag}$ nanocubes and $\mathrm{AuCl}_{2}{ }^{-}$or $\mathrm{AuCl}_{4}{ }^{-}$. Adv. Mater. 2008, 20, 2517-2522.

[21] Xiong, Y.; Wiley, B. J.; Chen, J.; Li, Z. -Y.; Yin, Y.; Xia, Y. Corrosion-based synthesis of single-crystal Pd nanoboxes and nanocages and their surface plasmon properties. Angew. Chem. Int. Ed. 2005, 44, 7913-7917.

[22] Kim, D.; Park, J.; An, K.; Yang, N. -K.; Park, J.-G.; Hyeon, T. Synthesis of hollow iron nanoframes. J. Am. Chem. Soc. 2007, 129, 5812-5813.

[23] Siekkinen, A. R.; McLellan, J. M.; Chen, J.; Xia, Y. Rapid synthesis of small silver nanocubes by mediating polyol reduction with a trace amount of sodium sulfide or 
sodium hydrosulfide. Chem. Phys. Lett. 2006, 432, 491496.

[24] Skrabalak, S. E.; Au, L.; Li, X.; Xia, Y. Facile synthesis of Ag nanocubes and Au nanocages. Nat. Protoc. 2007, 2, 2182-2190.

[25] Sieradzki, K. Curvature effects in alloy dissolution. J. Electrochem. Soc. 1993, 140, 2868-2872.

[26] Roosen, A. R.; Carter, W. C. Simulations of microstructural evolution: Anisotropic growth and coarsening. Physica A 1998, 261, 232-247.

[27] Wang, Z. L. Transmission electron microscopy of shapecontrolled nanocrystals and their assemblies. J. Phys. Chem. B 2000, 104, 1153-1175.

[28] Wiley, B. J.; Sun, Y.; Mayers, B.; Xia, Y. Shape-controlled synthesis of metal nanostructures: The case of silver. Chem. Eur. J. 2005, 11, 454-463.

[29] Tao, A.; Sinsermsuksakul, P.; Yang, P. Polyhedral silver nanocrystals with distinct scattering signatures. Angew. Chem. Int. Ed. 2006, 45, 4597-4601.

[30] Seo, D.; Yoo, C. I.; Park, J. C.; Park, S. M.; Ryu, S.; Song, $\mathrm{H}$. Directed surface overgrowth and morphology control of polyhedral gold nanocrystals. Angew. Chem. Int. Ed. 2008, 47, 763-767.

[31] Dick, K.; Dhanasekaran, T.; Zhang, Z.; Meisel, D. Size-dependent melting of silica-encapsulated gold nanoparticles. J. Am. Chem. Soc. 2002, 124, 23122317.

[32] Shi, H.; Zhang, L.; Cai, W. Composition modulation of optical absorption in $\mathrm{Ag}_{x} \mathrm{Au}_{1-x}$ alloy nanocrystals in situ formed within pores of mesoporous silica. J. Appl. Phys. 2000, 87, 1572-1574.

[33] McLellan, J. M.; Siekkinen, A.; Chen, J.; Xia, Y. Comparison of the surface-enhanced Raman scattering on sharp and truncated silver nanocubes. Chem. Phys. Lett. 2006, 247, 122-126.

[34] Wiley, B. J.; Im, S. H.; Li, Z. -Y.; McLellan, J. M.; Siekkinen, A.; Xia, Y. Maneuvering the surface plasmon resonance of silver nanostructures through shapecontrolled synthesis. J. Phys. Chem. B 2006, 110, 1566615675.

[35] Wiley, B. J.; Chen, Y.; McLellan, J. M.; Xiong, Y.; Li, Z. -Y.; Ginger, D. S.; Xia, Y. Synthesis and optical properties of silver nanobars and nanorice. Nano Lett. 2007, 7, 10321036.

[36] McLellan, J. M.; Li, Z. -Y.; Siekkinen, A. R.; Xia, Y. The SERS activity of a supported Ag nanocube strongly depends on its orientation relative to laser polarization. Nano Lett. 2007, 7, 1013-1017.

[37] Sherry, L. J.; Chang, S. H.; Schatz, G. C.; Van Duyne, R. P.; Wiley, B. J.; Xia, Y. Localized surface plasmon resonance spectroscopy of single silver nanocubes. Nano Lett. 2005, 5, 2034-2038.

[38] Chen, Y.; Munechika, K.; Ginger, D. S. Dependence of fluorescence intensity on the spectral overlap between fluorophores and plasmon resonant single silver nanoparticles. Nano Lett. 2007, 7, 690-696.

[39] Chen, Y.; Munechika, K.; Plante, I. J. -L.; Munro, A. M.; Skrabalak, S.; Xia, Y.; Ginger, D. S. Excitation enhancement of CdSe quantum dots by single metal nanoparticles. Appl. Phys. Lett. 2008, 93, 053106.

[40] Munechika, K.; Smith, J. M.; Chen, Y.; Ginger, D. S. Plasmon line widths of single silver nanoprisms as a function of particle size and plasmon peak position. J. Phys. Chem. C, 2007, 111, 18906-18911.

[41] Chen, Y.; Munechika, K.; Ginger, D. S. Bioenabled nanophotonics. MRS Bull. 2008, 33, 536-542. 